\title{
Extinguishing Stigma among Firefighters: An Examination of Stress, Social Support, and Help-Seeking Attitudes
}

\author{
Gemma M. Isaac, Marla J. Buchanan \\ Faculty of Education, The University of British Columbia, Vancouver, Canada \\ Email: marla.buchanan@ubc.ca
}

How to cite this paper: Isaac, G. M., \& Buchanan, M. J. (2021). Extinguishing Stigma among Firefighters: An Examination of Stress, Social Support, and Help-Seeking Attitudes. Psychology, 12, 349-373. https://doi.org/10.4236/psych.2021.123023

Received: November 23, 2020

Accepted: March 21, 2021

Published: March 24, 2021

Copyright (c) 2021 by author(s) and Scientific Research Publishing Inc. This work is licensed under the Creative Commons Attribution International License (CC BY 4.0).

http://creativecommons.org/licenses/by/4.0/

\begin{abstract}
Firefighters are exposed to highly stressful environments, often witnessing multiple traumatic events throughout their careers. The cumulation of stress and traumas firefighters are exposed to have left many in the profession with physical and psychological injuries, and with such injuries left untreated, can lead to lifelong suffering or suicide. The primary objectives for this research investigate firefighter occupational stress, peer support, and attitudes towards help-seeking for mental health in the hopes to fill in gaps understanding why firefighters continue to suffer in silence. Employing a mixed-methods research design, a survey questionnaire was collected from 254 firefighters from a large fire department in British Columbia, Canada. Consistent with the existing literature, findings suggest that the levels of peer support mitigated occupational stress, that is, those who reported higher levels of peer support also reported lower occupational stress levels. Firefighters provided information on what types of support they prefer according to the types and severity of stressors. Suggestions from the respondents provide information on how barriers to receiving help, such as stigma, may be addressed at the organizational level. Implications and recommendations for interventions addressing stigma and help-seeking amongst firefighters are discussed.
\end{abstract}

\section{Keywords}

Occupational Stress, Peer Support, Worker Attitudes

\section{Background}

After a growing number of suicides among firefighters across British Columbia, Canada, a call to action was organized by firefighter associations across the Province to address the stigma of help-seeking for those struggling with occupa- 
tional stress injuries and to promote mental health interventions within fire departments. While there has been increases of programs promoting mental health amongst firefighters, there remains a gap in firefighters' help-seeking behaviours resulting in many members who continue to suffer in silence. This article 1) discusses current research on stress, suicide, and attitudes towards psychological help-seeking within the fire service; 2 ) identifies firefighter sources of occupational stress, amount of perceived social support, attitudes towards seeking professional psychological help, and preferences and reasons for mental health providers; 3) explores issues and challenges of firefighter help-seeking and 4) and review respondent suggestions on policy considerations for fire departments and mental health professionals.

\section{Literature Review}

\subsection{Stress, Trauma, and Suicide in Firefighting}

Firefighting was named 2017's $2^{\text {nd }}$ most stressful job in the U.S. following the enlisted military personnel by the career information site Career Cast (2018), (Most stressful jobs of 2017). In general, firefighters are at higher risk of psychological problems due to the high and constant stress from exposure to highly traumatic events during their work (Bacharach, Bamberger, \& Doveh, 2008; Brown, Mulhern, \& Joseph, 2002; Carpenter et al., 2015; Gist \& Taylor, 2008; Henderson, Van Hasselt, Leduc, \& Couwels, 2016; Harris, Baloğlu, \& Stacks, 2002; O'Neill \& Rothbard, 2017; Kitt, 2009). In Canada, 1/3 of deaths by suicide are among people 45 - 59 years and is approximately 3 times higher among men compared to women (Government of Canada, 2018). The implications of suicide among this population would be worthwhile to examine in a predominantly male occupation such as firefighting.

The relationship between occupational stress and posttraumatic stress disorder (PTSD), places firefighters at increased risk of death by suicide compared to their civilian counterparts owing to higher rates of PTSD and substance use disorders (Henderson et al., 2016; Jakupcak et al., 2010; Jeannette \& Scoboria, 2008; Regehr, Hill, Knott, \& Sault, 2003; Hom, Stanley, Ringer \& Joiner, 2016). An important component of suicide prevention includes connecting at-risk individuals with mental health services. However, research has shown that a majority of suicide fatalities in the general population had contact with health care providers in the year prior to death, indicating that mental health problems often remain unidentified; representing a missed intervention opportunity (Ahmedani et al., 2014; Stanley, Hom, Hagab, \& Joiner, 2015).

A negative perception is frequently held of people with suicide ideation, labeling them as weak, selfish, or unable to cope with their problems (Carpiniello \& Pinna, 2017). Frey, Hans, and Cerel (2016) posit that the prevalence of suicide stigma expressed by social network members of individuals with previous suicidal behavior is a robust predictor of depression symptom severity. The complex relationship that exists between suicidal behavior and stigmatizing beliefs 
and attitudes is a growing area of study and one that has yet, to our knowledge, be investigated within the firefighter population. Studies by Berger, Levant, McMillan, Kelleher, and Sellers (2005) and Bulala (2013) illustrate how traditional masculinity definitions and negative stigma towards mental health services results in the underutilization of mental health services. Since firefighting is a male-dominant occupation and has a culture that upholds many traditional male values, we are curious to explore how being a firefighter poses a unique challenge as related to stigma for the respondents in this study.

The primary objectives for this research are to examine firefighter occupational stress, peer support, and attitudes towards help-seeking to gain a better understanding of the unique challenge's firefighter's face in connecting with effective mental health supports and interventions.

\subsection{Support for Firefighters}

A recent study on firefighter stress, supports and suicide ideation found a positive correlation between stress and suicidality, suggesting that firefighters are at risk for suicidal ideation, in part, because of their high levels of occupational stress (Carpenter et al., 2015). A number of studies postulate that social support appears to be a protective factor against suicide (Carpenter et al., 2015; Kimbrel et al., 2011; Meyer et al., 2012; Prati \& Pietrantoni, 2010). To gain a better understanding of the types of social supports firefighters deem to be helpful in mitigating their work-related stress, we investigate firefighters' perceived social support over the past 6 months. We then explore if a correlation between social support and occupational stress exists amongst our participants.

Firefighters exposure to critical incident stressors at the workplace, sometimes numerous exposures in one shift, are shown to be associated with the development of PTSD (Bonanno Galea, Bucciarelli, \& Vlahov, 2006; Meyer et al., 2012; Regehr et al., 2003; Slottje et al., 2008; Stanley et al., 2016), substance misuse (Bacharach et al., 2008), a decreased quality of life, reduced job satisfaction, high levels of work strain, functional impairment in various life spheres, lack of organizational support, lessened social support, interpersonal relationship difficulties, restricted range of affect, depressed mood, and social dysfunction (Bacharach et al., 2008; Lee, J., Lee, D., Kim, Jeon, \& Sim, 2017; North et al., 2002; Regehr et al., 2003).

In response to supporting firefighters after a critical incident exposure, peer led Critical Incident Stress Management (CISM) interventions are put into place at most fire departments mandating that a diffusion or a debrief take place after the traumatic incident. Studies have shown that the development of support networks such as peer support, management support, and debriefing sessions have improved coping capacities and decreased occupational stress and build resiliency among firefighters (Beaton \& Murphy, 1993; Haslam \& Mallon, 2003; Jeannette and Scoboria 2008; Regel 2007; Varvel et al., 2007). When given the option of a group CISD, firefighters' prefer peer led interventions with the belief 
that peers understand the experience better that non-firefighter counterparts (Jeannette \& Scoboria, 2008). Firefighters also identified that the type of support depended on the severity of the incident. Although a number of theories support the protective role of social support in the aftermath of critical incidents among first responders (Prati \& Pietrantoni, 2010), other theories point to social support following a traumatic event can exacerbate distress, creating exposure to the trauma (Stephens, Long, \& Miller, 1997).

Our research aims to contribute to the existing literature by building on firefighter preference for mental health interventions by asking firefighters reasons for seeking peer (including CISD interventions) or professional mental health supports. In identifying firefighters' most taxing occupational stressors and the preferences for what type of intervention they prefer; recommendations can be made at the operational level.

\subsection{Firefighters Attitudes and Stigma towards Help-Seeking}

There is a steady growth in the literature examining firefighters' general attitudes towards help-seeking (Bulala, 2013; Henderson et al., 2016; Hom et al., 2016; Norwood \& Rascati, 2012). The consensus is that the relatively closed culture of firefighting does not easily lend itself to talking to someone outside of the profession or work-family (Norwood \& Rascati, 2012). Studies examining law enforcements officers' attitudes towards seeking professional help illustrates that they held a more negative attitude toward seeking professional psychological help than the general population (Karaffa \& Tochkov, 2013).

Creation of successful mental health programs with firefighter buy-in requires examination of the unique firefighter occupational culture and the barriers they face when it comes to help-seeking. To our knowledge no empirical research has yet examined firefighters' attitudes towards seeking professional psychological help. Our research hopes to provide novel information regarding examining firefighters' attitudes toward seeking professional psychological help, as well as provider preferences for a variety of presenting problems.

The method of achieving these goals is in two-fold; beginning with a quantitative approach, firefighters identifies occupational stressors and support systems, attitudes about seeking psychological help and provider preferences for different issues. Next, firefighters are invited to provide comments on the subject of firefighter stress and accessing support as a means of providing this study with an in-depth understanding of the quantitative results.

\section{Method}

\subsection{Participants and Procedures}

Data was collected from February 2018 to March 2018 by means of an online survey questionnaire. Participants were recruited in a two-step process. First, an organization recruitment letter was distributed to the fire department chief and the union president explaining the goals of the study, potential risks and bene- 
fits, level of confidentiality and voluntariness of participation with a link to the electronic version of the research survey and a request for organizational consent to distribute the online survey to its membership. Second, the fire department sent out a recruitment letter of approximately 600 members and after the initial request for participation was sent, a reminder notice was sent two weeks later. Informed consent forms were attached to the front of the online survey questionnaire and the participants were required to indicate consent in order to access the survey. Eligibility criteria were explained (firefighter in the department, English language user). All contents of the survey package were coded numerically that provided a way to track the number of surveys distributed and received and ensured that multiple surveys were not completed by a single firefighter. The survey took approximately $1 / 2$ hour to complete. Ethics approval was obtained from the university Behavioural Research Ethics Board before the surveys were distributed and analyzed. In total, 258 responses were received using Qualtrics software. From these responses, 218 of the respondents completed all measures, which represent a response rate of $36.33 \%$ based on the possible 600 sample pool.

\subsection{Psychometric Tools}

The study consisted of seven self-administered questionnaires and one open ended question at the end of the survey for qualitative analysis of their experiences and opinions on firefighter occupational stress.

\subsubsection{The Sources of Occupation Stress Scale}

The Sources of Occupation Stress Scale revised 14-item version by Kimbrel et al. (2011), which was revised from Beaton and Murphy's (1993) 57-item self-report measure was utilized to examine firefighter-specific occupational stress. All items in the SOOS-14 employ a 5-point Likert-style response format ranging from "not stressed at all (1")" to "extremely stressed (5")" and measures occupational stress through a quantitative scale with a score between 14 and 70 . The SOOS-14 has demonstrated good reliability and validity in previous research with firefighters (Carpenter et al., 2015; Kimbrel et al., 2011). Internal consistency for the SOOS-14 in the current study was 0.79 .

\subsubsection{Firefighter Social Support Scale (SSS-FF)}

In this study the SSS-FF (Carpenter et al., 2015) was used to measure firefighter peer support. The 9-item measure also use a 5-point Likert-style response format ranging from "strongly disagree (1")" to "strongly agree (5")" using a scale with a score between 9 and 45. The SSS-FF was adapted from the Social Support Scale of the Deployed Risk and Resilience Inventory (King, L., King, D., Vogt, Knight, \& Samper, 2006) by Carpenter et al. (2015) to include only the items relevant to firefighters. The SSS-FF has been psychometrically validated with a Cronbach's alpha reliability that was found to be 0.81 (Carpenter et al., 2015). Special permission from the authors of the test was granted for this study. Cronbach's alpha 
for the SSS-FF in our study was 0.79 .

\subsubsection{Attitudes toward Seeking Professional Psychological Help-Short Form (ATSPPH-SF)}

Fischer and Farina's (1995) revised Attitudes Toward Seeking Professional Psychological Help-Short Form (ATSPPH-SF) revised from the Fischer and Turner (1970) 29-item measure of Attitudes Toward Seeking Professional Help for Psychological Disturbances was used to measure firefighters' general thoughts on seeking help from mental health providers, not employed directly by the Fire Department.

This ATSPPH-SF is made up of 10-items. For purposes of this study, scale attributes were modified slightly from the ATSPPH-SF and the direction of presentation reversed for clarity. That is, the attribute "agree" is modified to "strongly agree", "partly agree" is modified to "agree", "partly disagree" is modified to "disagree", and "disagree" is modified to "strongly disagree". The option "strongly disagree" is presented to the far left and "strongly agree" is presented to the far right. Items are rated on a 4-point Likert-type scale, where items 2, 4, 8,9 , and 10 are reversed scored. Therefore, scores on this scale could range from 10 to 40 . Scores are then summed together, with higher scores indicating more positive attitudes toward seeking professional help. Fischer and Farina's (1995) ATSPPH-SF was developed using a sample of undergraduate university student $(\mathrm{N}=389)$. The ATSPPH-SF correlates highly with the original ATSPPH $(\mathrm{r}=$ 0.87), and it has also demonstrated strong psychometric properties (Fischer \& Farina, 1995; Elhai, Schweinle, \& Anderson, 2008).

\subsubsection{Mental Health Provider Preference Questionnaire}

In order to build on the ATSPPH-SF and determine what type of mental health service firefighters prefer, a 14-item Mental Health Provider Preference Questionnaire was revised from a police officer measure devised originally by Karaffa and Tochkov (2013) and more recently by Wlodyka (2017) to fit the firefighter population. This questionnaire includes statements relating to different types of stressors firefighters could experience in the course of their careers, such as common work-related, interpersonal, and personal issues. Items refer to marriage and family issues, physical symptoms of stress, substance abuse, depression, organizational stress, task-oriented concerns, post-traumatic and critical incident stress, and suicide. The purpose of this measure is to assess firefighters' preferences for peer support versus a mental health professional for various issues. Higher scores reflect a greater preference for a professional mental health provider, and a lower score suggested a stronger preference for peer or CISM support within the Fire Department. Firefighters are asked to select their first preference and are limited in that they can only choose either peer or professional support for each issue.

\subsubsection{Reasons for Type of Mental Health Support}

Additionally, a four-item questionnaire on Mental Health Service Provider Pre- 
ference (adapted from Karaffa \& Tochkov, 2013; Wlodyka, 2017) was included to assess firefighters' reasons for seeking either peer or professional support and their thoughts about the frequency of seeing a professional. This questionnaire asks firefighters to select all items that are important to them.

A section for further comments was created for this survey to provide participants an opportunity to comment on the subject of firefighter stress and accessing peer and mental health support. The responses are analyzed using Braun and Clarke's (2006) six steps for thematic content analysis for psychological research.

The final questionnaire was designed to capture demographic information. It is developed specifically for this study. The content of the 6-items covers age, gender, level of education, years in firefighter service work, relationship status and rank.

We analyzed the data using statistical software. Descriptive statistics, including means and standard deviations, are reported for all measures. Analysis of variance (ANOVA) was used to determine the significance of differences between groups. Pearson's Product-Moment Coefficient correlational analysis was used to illustrate the relationship between attitudes towards seeking professional psychological help with various demographic factors. The descriptive analysis identified the most frequently mentioned stressors and was further subcategorized by the length of service groups. Qualitative data was analyzed and reported by themes.

\section{Results}

Two hundred and fifty-four online responses were received using the Qualtrics software. Two hundred eighteen (218) of the respondents completed all measures. Demographic characteristics are shown in Table 1. Respondents were primarily male at $96 \%$, with $4 \%$ of the sample being female. However, this is a good reflection of the actual $4 \%$ of female firefighters in the total sampled department.

\subsection{Occupational Stress}

The top 5 reported occupational stressors as measured by the SOOS-14 (Kimbrel et al., 2011, Carpenter et al., 2015) were as follows: disruption of sleep $(M=3.41$, $\mathrm{SD}=1.12)$; feelings of isolation from family due to work demands and stress ( $\mathrm{M}$ $=2.58, \mathrm{SD}=1.09)$; thoughts about past run(s) that have been particularly upsetting/disturbing $(\mathrm{M}=2.56, \mathrm{SD}=1.10)$; observing negative effect of stress on coworkers, (e.g., illness, alcohol abuse, and burn out) $(\mathrm{M}=2.56, \mathrm{SD}=1.04)$, and working with a substandard co-employee on emergency incidents or situations $(\mathrm{M}=2.50, \mathrm{SD}=1.21)$.

Sleep disturbances have been shown to be common in firefighters with PTSD symptomology (Haslam \& Mallon, 2003) and have been strongly linked to suicide (Bernert \& Joiner, 2007; Bjørngaard, Bjerkeset, Romundstad, \& Gunnell, 2011; Sjöström, Waern, \& Hetta, 2007). Similar to Carey, Al-Zaiti, Dean, Sessanna, \& Finnell (2011) study on sleep problems and quality of life for profes- 
sional firefighters, the respondents in our research identified sleep disturbances as their primary stressor $(\mathrm{M}=3.41, \mathrm{SD}=1.12)$. Interventions around firefighter sleep hygiene and further research on how to improve firefighters sleep would be an integral piece in supporting education to the mental health of firefighters.

Firefighters with 5 - 10 years of experience and those with more than 20 years of experience reported the highest level of occupational stress, while firefighters with less than 5 years of experience or 10 to 20 years on the job reported lower levels of occupational stress (see Figure 1). There was a significant effect of occupational stress on firefighters' years of service at the $p<0.05$ level for the three conditions $F(3,212)=4.91, p=0.003$.

\subsection{Stress and Support}

Frequency data of firefighter social support (see Table 2) collected from the SSS-FF illustrate respondents feel, in general, supported by their peers. One item, however, revealed that the respondents' sense of support was split when it came to discussing problems with peers. We then tested for statistical correlation between occupational stress and peer support using the Pearson correlation coefficient. Firefighter occupational stress and peers support were found to be moderately negative correlated, $r(236)=0.35, p<0.01$ (see Figure 2).

Table 1. Demographics.

\begin{tabular}{|c|c|c|c|c|c|c|c|}
\hline Variables & & $\mathbf{N}$ & Percent & Variables & & $\mathbf{N}$ & Percent \\
\hline \multirow{9}{*}{ Age } & $19-23$ & 3 & 1.4 & \multirow{4}{*}{ Service Range } & 0 - 5 years & 51 & 23.6 \\
\hline & $24-28$ & 22 & 10.1 & & $5-10$ years & 44 & 20.4 \\
\hline & $29-33$ & 36 & 16.5 & & $10-20$ years & 69 & 31.9 \\
\hline & $34-38$ & 32 & 14.7 & & $20+$ years & 52 & 24.1 \\
\hline & $39-43$ & 30 & 13.8 & \multirow{7}{*}{ Relationship } & Single & 23 & 10.6 \\
\hline & $44-48$ & 35 & 16.1 & & Separated & 5 & 2.3 \\
\hline & $49-53$ & 38 & 17.4 & & Divorced & 6 & 2.8 \\
\hline & $54-58$ & 17 & 7.8 & & Common Law & 31 & 14.3 \\
\hline & 59 and over & 5 & 2.3 & & Married & 144 & 66.4 \\
\hline \multirow{2}{*}{ Gender } & Male & 208 & 95.9 & & Partner & 7 & 3.2 \\
\hline & Female & 9 & 4.1 & & Other & 1 & 0.5 \\
\hline \multirow{7}{*}{ Education } & High school or equivalent & 11 & 5.0 & \multirow{7}{*}{ Rank } & Probationary firefighter & 8 & 3.7 \\
\hline & Some post-secondary & 81 & 37.2 & & Firefighter/EMT & 81 & 37.2 \\
\hline & Trades/Apprentice Certification & 55 & 25.2 & & Firefighter/Paramedic & 22 & 10.1 \\
\hline & Bachelors degree or equivalent & 52 & 23.9 & & Driver Engineer & 33 & 15.1 \\
\hline & Some graduate school & 11 & 5.0 & & Lieutenant & 32 & 14.7 \\
\hline & \multirow[t]{2}{*}{ Graduate school or higher } & 8 & 3.7 & & Captain & 37 & 17.0 \\
\hline & & & & & Battalion Chief & 5 & 2.3 \\
\hline
\end{tabular}




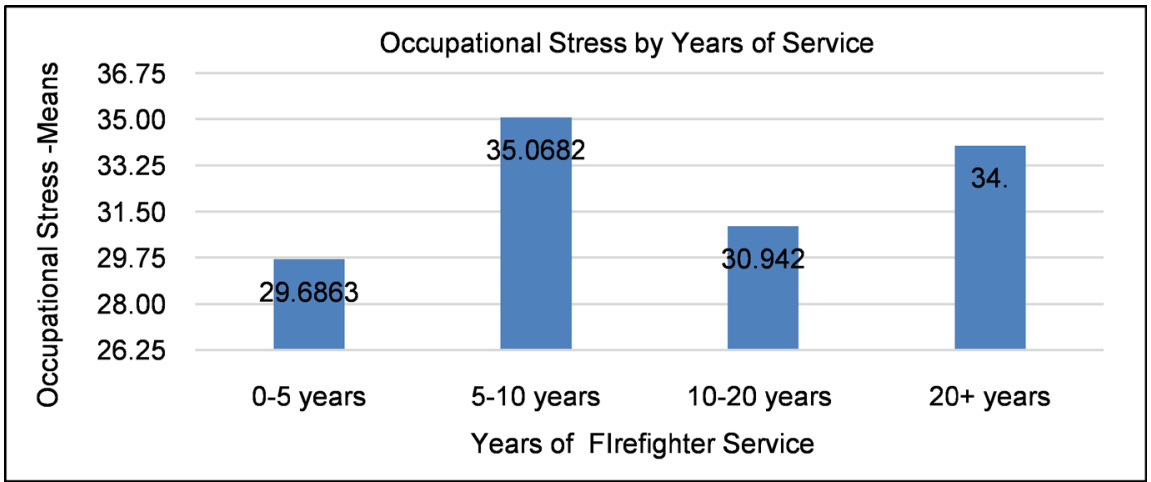

Figure 1. Firefighter occupational stress and years of firefighter service are measured as mean scores. Scored from a range of 14 - 70 where a higher number indicates higher levels of stress experienced on the job.

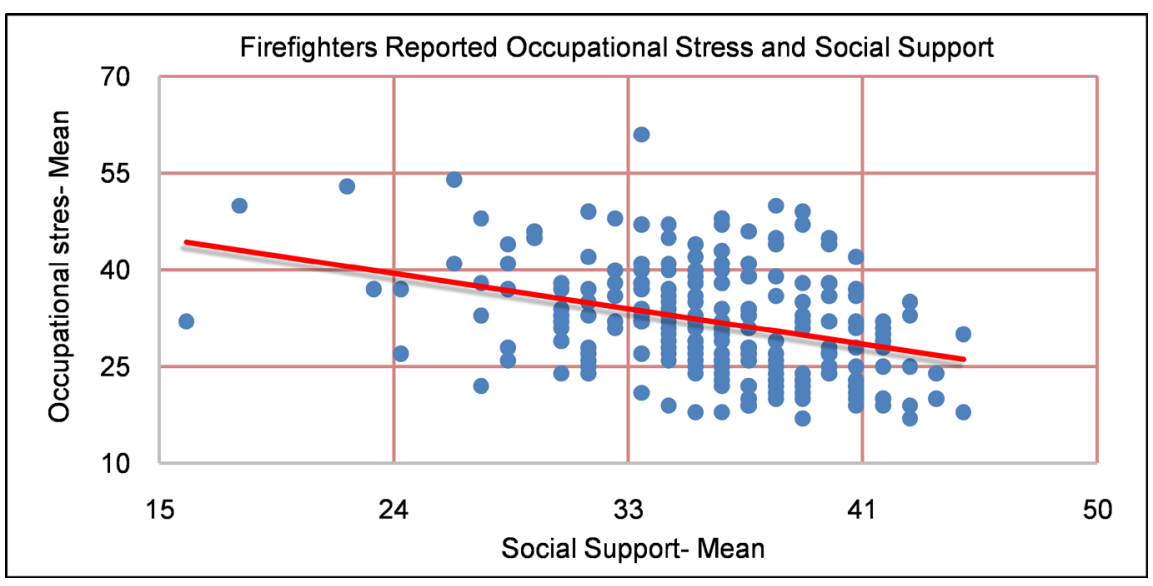

Figure 2. Firefighters reported occupational stress and social support. This figure illustrates a moderate negative correlation between firefighters reported occupational stress and social supports, that is, the greater the Social Support reported the less occupational stress experienced.

\subsection{Attitudes towards Seeking Professional Psychological Help}

An overall mean score of $(\mathrm{M}=29.63 \mathrm{SD}=4.75)$ was computed for the Fischer and Farina (1995) ATSPPH-SF scale (see Table 3). This score was compared to the normative group of college students established in the Fischer and Farina (1995) study using a one-sample t-test. The mean ATSPPH-SF score of firefighters in this sample was significantly higher than the control group $(M=$ 17.45, SD = 5.97). The internal consistency of the ATSPPH-SF using Cronbach's alpha was .84 and 1-month test-retest reliability was 0.80 for the normative sample. Using the current firefighter sample, internal consistency of the ATSPPH-SF was good, with a Cronbach's alpha of 0.80 . Using a one-sample t-test the mean was compared to 25 and the mean ATSPPH-SF score of the firefighter sample was $(\mathrm{M}=29.63 \mathrm{SD}=4.75) \mathrm{t}=17.76, p<0.0001$. Unlike Karaffa and Tochkov's (2013) study where police officers held a more negative attitude toward seeking professional psychological help than the general, the firefighters in our sample held a positive attitude towards seeking professional psychological help. 
Table 2. Respondents frequency of social support questionnaire.

\begin{tabular}{|c|c|c|c|c|c|}
\hline Types of support from Firefighter co-workers & $\begin{array}{l}\text { Strongly } \\
\text { Disagree }\end{array}$ & Disagree & $\begin{array}{l}\text { Neither agree } \\
\text { nor disagree }\end{array}$ & Agree & $\begin{array}{c}\text { Strongly } \\
\text { agree }\end{array}$ \\
\hline $\begin{array}{l}\text { You were carefully listened to and understood by } \\
\text { firefighters you worked with }\end{array}$ & 2.94 & 7.98 & 14.71 & 63.45 & 10.92 \\
\hline $\begin{array}{l}\text { Among your firefighter colleagues, there was someone who } \\
\text { made you feel better when you were feeling down. }\end{array}$ & 0.84 & 2.94 & 13.87 & 62.18 & 20.17 \\
\hline You had problems that you could discuss with other firefighters & 10.50 & 31.09 & 18.91 & 28.57 & 10.92 \\
\hline $\begin{array}{l}\text { Among your colleagues, there was someone you could } \\
\text { go to when you needed advice }\end{array}$ & 0.42 & 5.88 & 7.56 & 54.20 & 31.93 \\
\hline $\begin{array}{c}\text { There were people in the fire service you could talk to about } \\
\text { your experiences as a firefighter }\end{array}$ & 0.42 & 1.69 & 4.64 & 53.59 & 39.66 \\
\hline $\begin{array}{l}\text { The firefighters you knew respected the fact that you } \\
\text { were a firefighter }\end{array}$ & 0.42 & 0.84 & 16.03 & 54.85 & 27.85 \\
\hline You knew firefighters who would lend you money if you needed it & 0.84 & 3.78 & 25.21 & 49.16 & 21.01 \\
\hline $\begin{array}{l}\text { If you had been unable to did your daily chores at work, there was } \\
\text { someone in the firehall who would have helped you with these tasks }\end{array}$ & 2.94 & 2.94 & 5.46 & 55.04 & 33.61 \\
\hline $\begin{array}{l}\text { If you had been ill, there were other firefighters who would } \\
\text { have helped you }\end{array}$ & 0.84 & 0.84 & 3.38 & 35.86 & 59.07 \\
\hline
\end{tabular}

Note. Frequency reported in number of respondent percentages (\%).

Table 3. Mean and standard deviation of total attitudes towards seeking professional psychological help by years of service.

\begin{tabular}{cccc}
\hline Years of Service & $\mathrm{N}$ & $\mathrm{M}$ & S.D \\
\hline 1 - 5 years & 51 & 29.76 & 4.82 \\
5 - 10 years & 44 & 28.25 & 5.29 \\
10 - 20 years & 69 & 30.60 & 4.76 \\
20 or more years & 52 & 29.40 & 3.98 \\
Total & 216 & $\mathbf{2 9 . 5 0}$ & $\mathbf{4 . 7 2}$ \\
\hline
\end{tabular}

Note. $\mathrm{N}=$ number of years in service, $\mathrm{M}=$ mean value, $\mathrm{S} . \mathrm{D}=$ Standard Deviation.

Based on the studies illustrating men having more challenges with help seeking than women (Addis \& Mahalik, 2003; Berger et al., 2005), we examined if this was true in our sample and ran independent $t$-tests and found no significant difference in the scores for male firefighters $(M=29.50, S D=4.64)$ and female firefighters $(\mathrm{M}=31.11, \mathrm{SD}=6.88)$ attitudes; $\mathrm{t}(211)=-1.00, p=0.320$.

Notably, firefighters on the job from 5 to 10 years and 20 plus years who reported higher levels of occupational stress also reported less favorable attitudes towards seeking professional psychological help (see Figure 1 and Figure 3). Further investigation into this relationship will be developed with the qualitative data to provide suggestions for mental health interventions amongst these groups. 


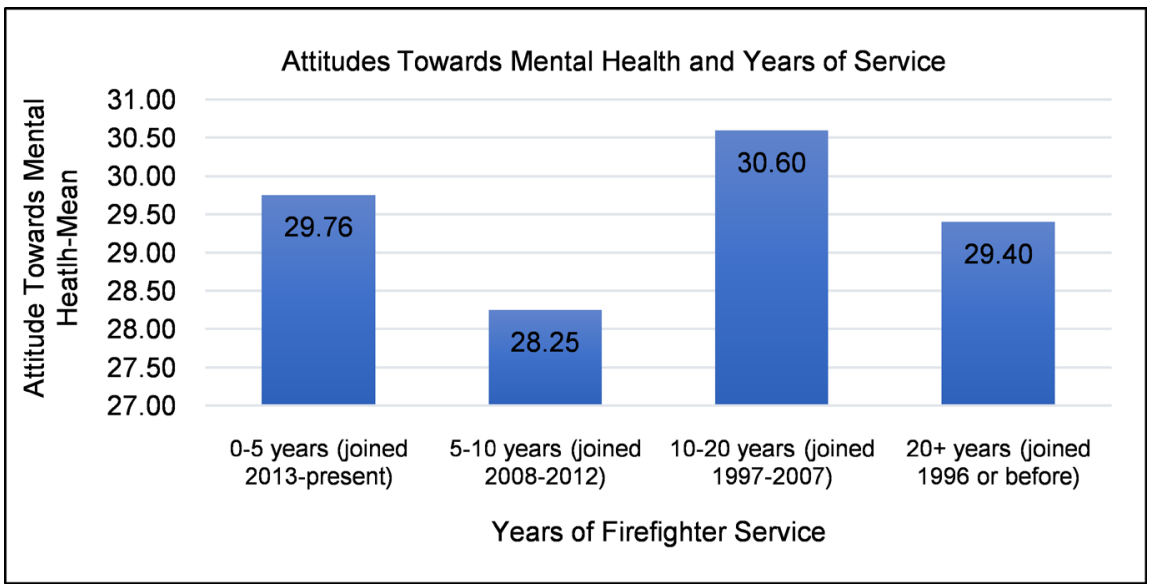

Figure 3. Attitudes towards mental health and years of firefighter service are measured as mean scores. Scored from a range of 10 - 40 where a higher number indicates a positive attitude towards help-seeking from a mental health professional for on-the-job stress.

Results from the Mental Health Provider Preference Questionnaire show that firefighters prefer fire department led peer or CISM support on four out of the seven items job-related stressors: 1) "If I experienced distress after a critical incident" ( $\mathrm{N}=133,82.43 \%) ; 2$ ) "If I were frustrated with organizational politics, colleagues, or supervisors" ( $\mathrm{N}=170,76.92 \%)$; 3) "If I were dealing with stress related to a particular event in which I was involved" ( $\mathrm{N}=159,72.28 \%)$; and 4$)$ "If I felt hopeless about the future of my career" ( $\mathrm{N}=123,55.71 \%)$. Conversely, the two items of workplace stress firefighters preferred a mental health professional over peer support were: 1) "If I were drinking alcohol excessively and this behavior was negatively affecting my ability to do my job" ( $\mathrm{N}=130,58.83 \%) ; 2)$ "If I suffered flashbacks nightmares of feelings of helplessness after experiencing or witnessing an event that involved serious injury or death (or threat of serious injury or death)" ( $\mathrm{N}=125,56.31 \%)$. One item, "If I had a difficult time controlling my emotions on the job and it was affecting my performance," received $50.45 \%$ in favor of mental health support and $49.55 \%$ in favor of peer support.

\subsection{Mental Health Provider Preference}

Regarding issues affecting personal lives, firefighters reported preference of help-seeking from a professional mental health provider for all, but one item presented affecting personal lives, that is, firefighters would rather see peer support, "If I felt as if the demands of the department were interfering in my relationships with friends and family" $(\mathrm{N}=99,55 \%)$. These results support previous studies findings of firefighter preference for peer guided mental health with the reason that fellow firefighters understand the unique job stressors affecting personal lives (Jeannette \& Scoboria, 2008). Respondents reported reasons for preference of a professional mental health provider for mental health-related issues was 1) they (professional mental health providers) have a higher level of training; 2) confidentiality; and 3) they have an independent opinion separate from 
the work environment.

\section{Qualitative Findings and Discussions}

Due to the volume of data collected, thematic content analysis was used inductively, and coding and analysis were primarily at the semantic level (Braun \& Clarke, 2006). We utilize reflexive thematic analysis to analyse the qualitative data to answer questions about firefighters' experiences around mental health and help seeking stigma. Following Braun and Clarke's 6-step analytic process, six main themes were found: 1) Access and Support; 2) Education and Cultural Awareness; 3) Stigma: Thoughts and Attitudes; 4) Operational Guidelines, Policy and Procedures; 5) Attitudes, Bullying and Lack of Trust Within the Department; and 6) Exposure to Trauma: Compounded and PTSD. Themes were chosen not only because of their prevalence across the majority of participants but also because they captured something important about the data in relation to the research questions (see Table 4).

\section{Theme 1: Access and Support (32/44 responses).}

Theme one, Access and Support, maps the positive and negative experiences with mental health access and support and describe participants expressed needs in this area where more could be done. Respondents acknowledged that programs and training had been put into place to support firefighters' mental health and wellness and they recognize the positive change in attitudes and programs supporting mental health in the department. However, others commented that although the department has made efforts to bring awareness to mental health and encourage a strong peer support system, there are still many challenges to receiving effective mental health interventions.

Some firefighters feel that psychological injuries and illnesses are not given "legitimate thought" and "sensitive consideration" when it comes to employee treatment and rehabilitation. Moreover, comments suggest that the current employment support they receive is ineffective resulting in feelings of frustration, humiliation, and lack of motivation to seek-help, stating that work managers were more focused on getting back to work, rather than treatment on recovery.

Table 4. Thematic analysis themes and response rate.

\begin{tabular}{lcc}
\hline \multicolumn{1}{c}{ Themes } & $\mathrm{N}$ & $\%$ \\
\hline Theme 1: Access and Support & 32 & 72.73 \\
Theme 2: Education and Cultural Awareness & 27 & 61.36 \\
Theme 3: Stigma: Thoughts and Attitudes & 13 & 29.55 \\
Theme 4: Operational Guidelines, Policy \& Procedures & 11 & 25.00 \\
Theme 5: Attitudes, Bullying and Lack of Trust Within the Department & 9 & 20.45 \\
Theme 6: Exposure to Trauma: Compounded and PTSD & 8 & 18.18
\end{tabular}

Note. $\mathrm{N}=$ frequency, $\%$ = percentage. 
Ten respondents provided proactive suggestions for better access and support such as professional mental health coverage under firefighter health benefits. It is less about the expense of treatment, than it is about acknowledging the unique demands of the job and the toll it takes on firefighters' mental, emotional, spiritual, and physical well-being.

Other suggestions regarding access and support include better signage at work regarding vetted contacts of mental health providers and educational opportunities for all parties (firefighters, management, work safe agencies, therapists), and having a mental health professional available in person to debrief firefighters after a serious incident. As shown in the mental health preference measure above, firefighters' reasons for professional support are that professional therapists are educated in and have and have more extensive knowledge around mental health than a volunteer peer support.

Theme 2: Education and Cultural Awareness (27/44 responses).

This theme represents a large umbrella of comments that encompass both psycho-educational aspects for those in the firefighter mental health field (firefighters, employers, peers, back to work agencies, and psychotherapists) and the importance of firefighter culture awareness across the board, especially with mental health professionals.

Respondents' preference for peer support is because they "get it", One participant suggests that a peer referred counsellor may mitigate the choice of having to choose between peer or professional support. Illustrating that the firefighting is a "closed culture", getting a referral from a peer lends to the idea that a trusted professional in some manner understands the unique firefighter culture. Respondents voiced that an educational piece is needed around understanding the different roles and therapeutic relationships of a professional mental health supporter versus peer support person to make a more informed decision when choosing types of support.

The major challenge firefighters reported with education is that training leaves them unprepared for tests because they are being taught by "inadequate teachers," causing undue occupational stress. Additionally, firefighters feel that back to work managers and employers need to be educated on firefighter mental health to understand the unique stressors they face, such as cumulative exposure to traumas, and the implications this has on their return-to-work programs.

Regarding seeking support from mental health professionals, a significant number of respondents noted the importance of mental health professionals having knowledge and training in firefighter culture and occupational awareness.

\section{Theme 3: Stigma-Thoughts and Attitudes (13/44 responses).}

Items in this theme are coded if participants mentioned attitudes towards seeking psychological help, the actual mention of the word "stigma", and any emotional charged words about judgment for seeking help. Two types of commentary surfaced: positive acceptance and negative challenges. The acceptances 
comments refer to remarks that attempt to normalize the stressors of the job as being the "nature of the job" and that debriefs aren't always warranted as "each firefighter copes differently". This comment supports the mixed findings on the effectiveness of CISD as noted above.

Stigma surrounding mental illness and mental health services is considered to be a contributing factor for men not seeking help (Vogel, Wade, \& Ascheman, 2009). In this stigma definition, mental illness is associated with being perceived as different, dangerous, or unacceptable (Bulala 2013). Bulala (2013) describes another type of stigma called self-stigma, where the individual perceives the act of seeking professional help for distress as a threat to their self-worth and as a character flaw (Vogel, Wade, \& Haake, 2006). According to Thurnell-Read and Parker (2008), a firefighter's identity is tied to emotional strength, physical and technical competence and collective understandings of risk and responsibility. Where self-stigma exists, help-seeking would threaten a firefighter's occupational identity (Henderson et al., 2016; Norwood \& Rascati, 2012). Respondents' comments support this theory stating that a barrier for mental health help-seeking is due to "pride" or "fear of what others think". Others noted that stigma kept them from accessing support as they did not want to be "perceived as weak". Consequently, firefighters are choosing to "suffer in silence".

Theme 4: Operational Guidelines, Policy \& Procedures (11/44 responses).

Theme four includes comments that mentioned challenges and suggestions regarding current operational guidelines, policies and procedures that were not being met or executed efficiently are presented.

Suggestions on how to introduce new policies and procedures following critical incidents illustrates how firefighters in this sample are ready for a change and seek both peers and mental health professionals' support for mental health. Recommendations include: 1 ) debriefings must be mandatory after critical incident calls; 2) senior officers that decide against CISM debriefing for their crews from incidents that fall under the mandatory criteria should be suspended without pay automatically; and 3) that professional mental health check-ups become mandatory practice.

Theme 5: Attitudes, Bullying and Lack of Trust within the Department (9/44 responses).

All nine comments in this theme fell into the category of current challenges faced by firefighters, feelings of frustration, bullying, lack of trust, and general discontent with workplace support. One participant shared

It is a poisonous work environment that is only good for the majority of white males. I have never been more depressed and isolated in my life; I know I'm not the only one. My PTSD is more so from my fears of the co-workers and little from the actual calls... they will exaggerate and even flat out lie regarding something that happened in the hall and they will spread it like wildfire.

The comments provide insight to why the SSS-FF measure illustrated firefighters were split about discussing problems with peers, lending to the assump- 
tion that firefighters may not easily feel like they can seek-help amongst peers even with issues closely related to work related stress. Sub-themes emerged in this category of lack of trust regarding confidentiality within the department, gossip within the department often stemming from higher-ranking officers, and bullying and intimidation tactics from co-workers.

This theme may also shed some light on why firefighters in the current study who have been on the job for $5-10$ years and 20 years or more are the cohorts reporting higher on the job stress yet score lower on seeking psychological help. Addis and Mahalik's (2003) study on men, masculinity and the context of help seeking support posit that men are less likely to seek help for problems that they deem unusual, especially if these issues are perceived as central to his identity. Furthermore, if the group of men they seek help from endorse the norms of self-reliance or feels like the social group would reject him that be a threat to their autonomy. For a firefighter in a hyper masculine and para-military hierarchal career, 5 - 10 years on the job may be the threshold where they feel too far into the career to not be able to attribute being a "probie" (probationer) any longer to ask for help and not wanting to seem green on the job. For the experienced firefighter who's been subjected to the "suck it up" mentality for over a $1 / 3$ of their lives, and now has an identity of being a high rank officer and not wanting to appear weak amongst their subordinates, asking for help challenges their firefighter identity. As a result, efforts should be made to combat the persisting stigmatizing attitudes of firefighters displayed toward mental disorders and suicide especially amongst the cohort, by normalizing help-seeking for all types of work stress and injuries, including mental health.

Theme 6: Exposure to Trauma-Multiple Traumas and PTSD (8/44 responses).

Theme six encompasses comments shared about multiple exposures to trauma, how the work has negatively impacted them, and the effects of working so closely with death daily. Although this theme had the least responses, we found them compelling given their depiction of "what is left within them" at the end of the day. All eight comments focused on the challenges of exposure to trauma. Comments like compound stress in addition to working at a toxic environment create a firefighter's "breaking point". We also get a glimpse of how work stress affects the home life as two comments noted how the job "hardened me at the cost to my family". This theme demonstrates how important consistent mental health throughout a fire fighters' career is due to the number of trauma exposures experienced, and that support needs to extend beyond the firefighter and also be provided to their family members.

\section{Conclusion}

To understand why firefighters are suffering in silence, the study first examines the severity of what on the job stress affects them on a regular basis, the amount of perceived social support firefighters receives from peers, attitudes seeking 
professional psychological help, and preference and reasons for types of mental health providers. We then compared the data from different questionnaires to see if a correlation from one variable to the next, for example, severity of work stress to years of service. Furthermore, we looked for developing themes among the firefighters' comments to give us a deeper understanding for the responses given in the quantitative section.

The current study supports Jeannette and Scoboria's (2008) findings that firefighters often choose peer supports when it comes to work related stress as they feel them "get-it". However, comments from the respondents also painted a picture that trust, gossip, and confidentiality were concerns of help-seeking amongst peers and for that reason would consider seeing a confidential professional mental health practitioner for the same issues. With more personal matters such as marriage or mental health firefighters' reason that professional supports are better educated in this realm. Participants also commented that they would seek support for both personal and work issues from mental health professionals who were more competent in firefighting culture. One firefighter describes it would be helpful to see a therapist who understood the "sights, sounds, and smells of the job." In addition to mental health providers, firefighters suggest that administrators and back to work managers who understand the unique firefighter culture are key to promoting help-seeking behaviour.

The survey results and comments by participants in the current study not only reflect all of the major themes regarding occupational stressors, peer supports, and attitudes towards psychological help for firefighters, they also add significant detail to the types of mental health supports preferred by this cohort. This research brings together previously identified issues such as stigma of help-seeking amongst firefighters as well as novel themes of access to mental health supports for firefighters, such as targeting mental health interventions for firefighters at the mid and end of their career, and supporting firefighter family members mental health, to form a comprehensive picture of how being a firefighter impacts the process of connecting with support for their mental health needs.

To our knowledge, this is one of the first studies to provide an in-depth examination of firefighters' preferences for the type of mental health supports addressing firefighter work and personal issues. The findings contribute to an understanding of the firefighter culture according to a sizeable, urban, Canadian population of professional firefighters and their attitudes towards help-seeking. The study also points to the importance of firefighter culture competency amongst administrators and mental health professionals in promoting help-seeking assistance for mental health issues. By combining quantitative and qualitative methods in this study, we sought to address gaps in the extant literature by understanding the help-seeking attitudes of firefighters, and to gather information from their vantage point on how to decrease stigma around-help seeking so that firefighters do not feel like they have to suffer in silence. The firefighters who participated in this research provided much needed future direction that can in- 
form fire department administrators and influence organizational guidelines for workplace health and safety to extinguish the stigma around help seeking for mental health promoting that asking for help is not a sign of weakness, rather one of resilience.

\section{Implications}

The findings of this study have several implications for professionals involved in working with firefighters. The quantitative and qualitative data illustrate firefighters' preference for help seeking towards someone who understands the firefighting culture and nature of the traumas they are exposed to. Counsellors competent with firefighter culture are regarded as a preference over other counsellors as one firefighter suggested that counsellors go on a ride-along to experience and understand the sights sounds, and smells which firefighters face daily.

Trust and confidentiality were two other factors firefighters expressed when choosing mental health support. Some firefighters commented on the "horrible" experiences they have when attempting to connect with supports at the workplace with peers, employers, or back to work agencies, even with mental health professionals. Additionally, comments highlighting stigma around mental health at the workplace lend to promoting interventions that normale help-seeking behaviours amongst all ranks in the department, and that mandatory confidential mental health check-ins may be one of those interventions that can model non-judgmental help-seeking within the department.

Because firefighters are exposed to trauma throughout their careers, sometimes multiple traumas in one shift, it is important to provide interventions that firefighters can utilize with day to day coping strategies that they can practice on the job to maintain their health and wellness. Moreover, comments regarding the complexity of multiple personal and work stressors had respondents suggesting that a holistic approach, including physical, emotional, spiritual, and cognitive wellbeing needs to be considered in supporting mental health. Program planning may include interdisciplinary resources to support firefighters, such as recreational and physical therapies, outdoor therapy, family therapy, music therapy, Chaplain Support, and financial planning supports.

\section{Future Research Directions}

Though this study is believed to be the first in Canada to examine firefighter occupational stress, peer supports, and attitudes towards seeking professional psychological help; future research identifying further behavioural health initiatives supporting firefighters after their careers are needed. As our study finds, firefighters in the end part of their career are experiencing less support, and the implications of retiring from a career that is like a second family and home, is a big life transition requiring support. Furthermore, firefighters reported the most stressful event is sleep disruption and the research supporting how sleep disorders can lead to poor mental health, research on optimizing firefighter sleep 
would be worthwhile.

Other firefighters mentioned that peer support was important to them; however, trust and confidentiality within the department seemed to be a challenge. Peer supports often lack advanced mental health training to the extent of a registered or certified counsellor or psychologist, and most departments have not implemented a practice of who supports the peer supporter. Further, research in identifying appropriate peer support, possibly even recruiting retired firefighters, who understand the culture and nature of the calls, yet have a separation from being active on the floor and seeing their peers after providing support, may be an option for future peer support programs. Lastly, with the rollout of new firefighter occupational awareness training for therapists, research on therapeutic relationships with an occupationally aware trained therapist in comparison to a therapist without the training may provide some insight if this additional education supports the idea of being better understood by a mental health professional.

\section{Limitations}

This survey was distributed to only one large firefighting department in the Lower Mainland of B.C. This particular department has been the recipient of several surveys over the past few years, with one particular survey containing the same occupational stress measure (SOOS-14). Participation in the study was voluntary, which likely led to participants declining the survey if they thought they had already completed this questionnaire before. Furthermore, this was only one large department in the Lower Mainland; the research results may vary from department to department depending on behavioural health support initiatives.

\section{Recommendations for Firefighter Organizations}

Based on this study's findings, the following recommendations are suggested to enhance access to psychological care within fire organizations.

Recommendation 1: Firefighter Departments should consider reviewing their health benefit plans with their employers to include registered clinical counsellors in addition to registered clinical psychologists.

Rationale: A number of specific requests were made to see occupationally aware counselling therapists and counsellors recommended by peers. Generally, there are long waits to see a registered psychologist, whereas counselling therapists are more accessible. Some firefighters may also have a pre-existing relationship with counsellors before entering the department. As one firefighter mentioned, other creative art therapies (music therapy, yoga therapy, drama therapy) should also be considered in broadening the pool of accessible behavioural health therapies. That could lead to timelier interventions and much-needed care for firefighters' overall health and wellness.

Recommendation 2: Firefighter Organizations should consider reviewing their extended health benefit plans to add group counselling.

Rationale: Firefighters CISM team support defusing as the most important 
organizational support after a critical incident. They also noted that the top two reasons for contacting peer support are understanding the issues and trusting a fellow firefighter. In comparison, there have been major criticisms regarding trust and confidentiality amongst peers. A mental health professional lead counselling group would provide a safe and confidential environment that continues on the strengths of group processing beyond the brevity of short-term critical incident group defusing and debriefings.

Recommendation 3: Provide education, both theoretical and experiential (ride-along, hall visits, table talks) for mental health providers on the occupational awareness of firefighters.

Rationale: Firefighters in this study showed positive attitudes towards mental health service providers, but they also noted that they have a higher level of training in mental health issues. However, the biggest challenge participants noted was finding counsellors who "get it" and the importance it was to speak with a counsellor who understood the culture and challenges of the job.

Recommendation 4: Continue to conduct psycho-educational training on firefighter stress and coping mechanisms throughout a firefighter's career, starting in the firefighter training and continuously every 2 - 3 years as part of a firefighter training cycle.

Rationale: This training will encourage conversations about signs of burnout, occupational stressors, and PTSD. It will also provide an opportunity to remind firefighters about psychological services available to them and their entitlements within their benefits.

Recommendation 5: Provide education for firefighter spouses and close family members (parents or siblings) at various stages of a firefighter's career on the effects of occupational stress, signs, and symptoms to look out for, as well as family resources.

Rationale: Social supports, including family, have been well documented as mitigating the effects of PTSD. Corneil, Beaton, Murphy, Johnson, \& Pike (1999) found that marriage was a protective factor against PTSD in the United States sample.

Recommendation 6: Provide a transition program for retiring firefighters.

Rationale: Within this study, firefighters with 20 or more years of service reported the second highest mean stress levels. A firefighter's direct comment also noted that it was in this stage when they were not as active on the floor with calls that they had time to ruminate and think about the past calls. Many of these firefighters are in the later stages of their career and nearing retirement. A program that would allow them to address some of the cumulative trauma they have experienced before exiting firefighting and losing extended health benefits would likely be beneficial.

Recommendation 7: Educate supervisors (Chiefs, Captains, Lieutenants) on signs and symptoms to look out for their subordinates. Encourage supervisors to check-in with their members regularly and especially after involvement in criti- 
cal incidents. After a critical incident table talk should be initiated, and at that time, a confidential vote such as everyone marking yes or no on a piece of paper, and placed in a box, opened up, and tallied for a CISD should be taken.

Rationale: Given the paramilitary hierarchy that exists within the firefighting culture and the fear of lower ranking firefighters fearing to speak up for support out of fear up appearing weak, it does not help when "Senior officers decide against CISM debriefings for their crews from incidents that fall under the mandatory criteria". Therefore, training Chiefs to become adequate "with the care of their employee's mental health" would be a shift in the current attitudes towards seeking mental health support at work.

Recommendation 8: Adequately screen staff and train; CISM peer support teams have peer nominations on CISM peer support teams.

Rationale: CISM team peer support was identified by $71 \%$ of participants as the most important department-led initiative following a critical incident. Despite strong support for the program, concerns were raised by firefighters within this study, "It is very hard to maintain confidentiality with all of our CISM team members." This highlights the importance of a selective screening process and a confidentiality agreement among its members. Another firefighter identified, "I am not a fan of CISM and peers support... Firefighters are not trained professionals who have gone to school for over four years." This response highlights the need for appropriate standardized peer support training and psychoeducation on the floor to identify the role of peer support versus mental health provider support to provide an effective and credible service for firefighter membership.

Recommendation 9: Educate workplace injury insurance agencies and back to work employers on mental health and occupational awareness.

Rationale: Firefighters described various reasons why employers and agencies working with the employers of employee occupational health and safety and return to were "horrible to deal with" and would "rather use a sick day" than deal with such agencies. Providing occupational awareness regarding the unique psychological stress firefighters face as a part of a workplace injury and illness could help firefighters rehabilitate faster and return to work effectively and efficiently.

Recommendation 10: Organizations should maintain an up-to-date list of psychological service providers with occupational awareness covering various geographical locations based on where firefighters are living. This information should be easily and anonymously accessible. CISM team members could check-in regularly with mental health professionals to adjust recommendations as needed based on wait times and firefighter feedback.

Rationale: The need for simple and anonymous access to information on psychological providers competent with firefighter occupational awareness was highlighted in the study. Furthermore, there needs to be additional support for the volunteer members who take on the role of peer supports and CISM members as 
they have to take on their peers' "mental load".

Recommendation 11: Consider implementing mandatory counselling sessions every one to two years for active firefighters.

Rationale: To overcome the stigma of seeing a mental health professional, mandatory counselling may be a means of overcoming that barrier in enhancing firefighters' psychological health. Mandatory counselling following a serious critical incident was supported by $33 \%$ of the participants and $24 \%$ of participants thought it would be good for firefighter members to attend mandatory counseling at least once a year.

Recommendation 12: Consider adopting the Canadian national standard for psychological health in the workplace or connecting with the Veteran Affairs Canada (VAC) a national network of mental health supports for first responders.

Rationale: The implementation of the Canadian National Standard would provide an overarching framework and direction for firefighting departments to ensure that various psychological supports are implemented and maintained. In connecting with VAC, a relationship can be built to support nationwide first responders who "witness some horrific scenes that would rival a war zone at times." These national bodies that support behavioural health would help extinguish the stigma around mental health and provide a message to all the firefighter members that mental health is supported at the highest levels.

\section{Acknowledgments}

The authors are grateful for the support and participation of IAFF Local 18 members, the Vancouver Fire and Rescue Services Chief Darrell Reid, and IAFF Local 18 President Rob Weeks for recognizing and supporting the importance of behavioural health within the department and endorsing this study to the members.

\section{Conflicts of Interest}

The authors declare no conflicts of interest regarding the publication of this paper.

\section{References}

Addis, M. E., \& Mahalik, J. R. (2003). Men, Masculinity, and the Contexts of Help Seeking. American Psychologist, 58, 5-14. https://doi.org/10.1037/0003-066X.58.1.5

Ahmedani, B. K., Simon, G. E., Stewart, C., Beck, A., Waitzfelder, B. E., Rossom, R., Solberg, L. I. et al. (2014). Health Care Contacts in the Year before Suicide Death. Journal of General Internal Medicine, 29, 870-877. https://doi.org/10.1007/s11606-014-2767-3

Bacharach, S. B., Bamberger, P. A., \& Doveh, E. (2008). Firefighters, Critical Incidents, and Drinking to Cope: The Adequacy of Unit-Level Performance Resources as a Source of Vulnerability and Protection. Journal of Applied Psychology, 93, 155-169. https://doi.org/10.1037/0021-9010.93.1.155

Beaton, R., \& Murphy, S. (1993). Sources of Occupational Stress among Firefighter/EMTs 
and Firefighter/Paramedics and Correlations with Job-Related Outcomes. Prehospital and Disaster Medicine, 8, 140-147. https://doi.org/10.1017/S1049023X00040218

Berger, J. M., Levant, R., McMillan, K. K., Kelleher, W., \& Sellers, A. (2005). Impact of Gender Role Conflict, Traditional Masculinity Ideology, Alexithymia, and Age on Men's Attitudes toward Psychological Help Seeking. Psychology of Men and Masculinity, 6, 73-78. https://doi.org/10.1037/1524-9220.6.1.73

Bernert, R. A., \& Joiner, T. E. (2007). Sleep Disturbances and Suicide Risk: A Review of the Literature. Neuropsychiatric Disease and Treatment, 3, 735-743. https://doi.org/10.2147/NDT.S1248

Bjørngaard, J. H., Bjerkeset, O., Romundstad, P., \& Gunnell, D. (2011). Sleeping Problems and Suicide in 75,000 Norwegian Adults: A 20 Year Follow-Up of the HUNT I Study. Sleep, 34, 1155-1159. https://doi.org/10.5665/SLEEP.1228

Bonanno, G. A., Galea, S., Bucciarelli, A., \& Vlahov, D. (2006). Psychological Resilience after Disaster: New York City in the Aftermath of September 11th Terrorist Attack. Psychological Science, 17, 181-186. https://doi.org/10.1111/j.1467-9280.2006.01682.x

Braun, V., \& Clarke, V. (2006). Using Thematic Analysis in Psychology. Qualitative Research in Psychology, 3, 77-101. https://doi.org/10.1191/1478088706qp063oa

Brown, J., Mulhern, G., \& Joseph, S. (2002). Incident-Related Stressors, Locus of Control, Coping, and Psychological Distress among Firefighters in Northern Ireland. Journal of Traumatic Stress, 15, 161-168. https://doi.org/10.1023/A:1014816309959

Bulala, J. (2013). The Relationship between the Utilization of Mental Health Services, Coping Mechanisms, and Reputation in Male Firefighters. Alhambra, CA: Alliant International University.

Career Cast (2018). Most Stressful Jobs of 2017. https://www.careercast.com/jobs-rated/most-stressful-jobs-2017?page=1

Carey, M. G., Al-Zaiti, S. S., Dean, G. E., Sessanna, L., \& Finnell, D. S. (2011). Sleep Problems, Depression, Substance Use, Social Bonding, and Quality of Life in Professional Firefighters. Journal of Occupational and Environmental Medicine, 53, 928-933. https://doi.org/10.1097/JOM.0b013e318225898f

Carpenter, G. S., Carpenter, T. P., Kimbrel, N. A., Flynn, E. J., Pennington, M. L., Cammarata, C., Gulliver, S. B. et al. (2015). Social Support, Stress, and Suicidal Ideation in Professional Firefighters. American Journal of Health Behavior, 39, 191-196. https://doi.org/10.5993/AJHB.39.2.5

Carpiniello, B., \& Pinna, F. (2017). The Reciprocal Relationship between Suicidality and Stigma. Frontiers in Psychiatry, 8, 35. https://doi.org/10.3389/fpsyt.2017.00035

Corneil, W., Beaton, R., Murphy, S., Johnson, C., \& Pike, K. (1999). Exposure to Traumatic Incidents and Prevalence of Posttraumatic Stress Symptomatology in Urban Firefighters in Two Countries. Journal of Occupational Health Psychology, 4, 131-141. https://doi.org/10.1037/1076-8998.4.2.131

Elhai, J. D., Schweinle, W., \& Anderson, S. M. (2008). Reliability and Validity of the Attitudes toward Seeking Professional Psychological Help Scale-Short Form. Psychiatry Research, 159, 320-329. https://doi.org/10.1016/j.psychres.2007.04.020

Fischer, E. H., \& Farina, A. (1995). Attitudes towards Seeking Professional Psychological Help: A Shortened Form and Consideration for Research. Journal of College Student Development, 36, 368-373. https://doi.org/10.1037/t05375-000

Fischer, E. H., \& Turner, J. I. (1970). Orientations to Seeking Professional Help: Development and Research Utility of an Attitude Scale. Journal of Consulting and Clinical Psychology, 35, 79-90. https://doi.org/10.1037/h0029636 
Frey, L. M., Hans, J. D., \& Cerel, J. (2016). Perceptions of Suicide Stigma: How Do Social Networks and Treatment Providers Compare? Crisis: The Journal of Crisis Intervention and Suicide Prevention, 37, 95-103. https://doi.org/10.1027/0227-5910/a000358

Gist, R., \& Taylor, V. H. (2008). Occupational and Organizational Issues in Emergency Medical Services Behavioral Health. Journal of Workplace Behavioral Health, 23, 309-330. https://doi.org/10.1080/15555240802243120

Government of Canada (2018). Suicide in Canada: Key Statistics (Infographic). https://www.canada.ca/en/public-health/services/publications/healthy-living/suicide-ca nada-key-statistics-infographic.html

Harris, M. B., Baloğlu, M., \& Stacks, J. R. (2002). Mental Health of Trauma-Exposed Firefighters and Critical Incident Stress Debriefing. Journal of Loss and Trauma, 7, 223-238. https://doi.org/10.1080/10811440290057639

Haslam, C., \& Mallon, K. (2003). A Preliminary Investigation of Posttraumatic Stress Symptoms among Firefighters. Work \& Stress, 17, 277-285.

https://doi.org/10.1080/02678370310001625649

Henderson, S. N., Van Hasselt, V. B., Leduc, T. J., \& Couwels, J. (2016). Firefighter Suicide: Understanding Cultural Challenges for Mental Health Professionals. Professional Psychology: Research and Practice, 47, 224-230. https://doi.org/10.1037/pro0000072

Hom, M. A., Stanley, I. H., Ringer, F. B., \& Joiner, T. E. (2016). Mental Health Service Use among Firefighters with Suicidal Thoughts and Behaviors. Psychiatric Services, 67, 688-691. https://doi.org/10.1176/appi.ps.201500177

Jakupcak, M., Vannoy, S., Imel, Z., Cook, J. W., Fontana, A., Rosenheck, R., \& McFall, M. (2010). Does PTSD Moderate the Relationship between Social Support and Suicide Risk in Iraq and Afghanistan War Veterans Seeking Mental Health Treatment? Depression and Anxiety, 27, 1001-1005. https://doi.org/10.1002/da.20722

Jeannette, J. M., \& Scoboria, A. (2008). Firefighter Preferences Regarding Post-Incident Intervention. Work and Stress, 22, 314-326. https://doi.org/10.1080/02678370802564231

Karaffa, K. M., \& Tochkov, K. (2013). Attitudes toward Seeking Mental Health Treatment among Law Enforcement Officers. Applied Psychology in Criminal Justice, 9, 75-99.

Kimbrel, N. A., Steffen, L. E., Meyer, E. C., Kruse, M. I., Knight, J. A., Zimering, R. T., \& Gulliver, S. B. (2011). A Revised Measure of Occupational Stress for Firefighters: Psychometric Properties and Relationship to Posttraumatic Stress Disorder, Depression, and Substance Abuse. Psychological Services, 8, 294-306. https://doi.org/10.1037/a0025845

King, L. A., King, D. W., Vogt, D. S., Knight, J., \& Samper, R. E. (2006). Deployment Risk and Resilience Inventory: A Collection of Measures for Studying Deployment-Related Experiences of Military Personnel and Veterans. Military Psychology, 18, 89-120. https://doi.org/10.1207/s15327876mp1802_1

Kitt, L. R. (2009). Breaking the Silence: Insights into the Impact of Being a Firefighter on Men's Mental Health. Unpublished Doctoral Dissertation, Vancouver: The University of British Columbia.

Lee, J. H., Lee, D., Kim, J., Jeon, K., \& Sim, M. (2017). Duty-Related Trauma Exposure and Posttraumatic Stress Symptoms in Professional Firefighters. Journal of Traumatic Stress, 30, 133-141. https://doi.org/10.1002/jts.22180

Meyer, E. C., Zimering, R., Daly, E., Knight, J., Kamholz, B. W., \& Gulliver, S. B. (2012). Predictors of Posttraumatic Stress Disorder and Other Psychological Symptoms in Trauma-Exposed Firefighters. Psychological Services, 9, 1-15. 
https://doi.org/10.1037/a0026414

North, C. S., Tivis, L., McMillen, J. C., Pfefferbaum, B., Spitznagel, E. L., Cox, J., Smith, E. M. et al. (2002). Psychiatric Disorders in Rescue Workers after the Oaklahoma City Bombing. The American Journal of Psychiatry, 159, 857-859. https://doi.org/10.1176/appi.ajp.159.5.857

Norwood, P. J., \& Rascati, J. N. (2012). Recognizing and Combating Firefighter Stress. Fire Engineering, 87-89.

O’Neill, O. A., \& Rothbard, N. P. (2017). Is Love All You Need? The Effects of Emotional Culture, Suppression and Work-Family Conflict on Firefighter Risk-Taking and Health. Academy of Management Journal, 60, 78-108. https://doi.org/10.5465/amj.2014.0952

Prati, G., \& Pietrantoni, L. (2010). The Relation of Perceived and Received Social Support to Mental Health among First Responders: A Meta-Analytic Review. Journal of Community Psychology, 38, 403-417. https://doi.org/10.1002/jcop.20371

Regehr, C., Hill, J., Knott, T., \& Sault, B. (2003). Social Support, Self-Efficacy and Trauma in New Recruits and Experienced Firefighters. Stress and Health, 19, 189-193. https://doi.org/10.1002/smi.974

Regel, S. (2007). Post-Trauma Support in the Workplace: The Current Status and Practice of Critical Incident Stress Management (CISM) and Psychological Debriefing (PD) within Organizations in the UK. Occupational Medicine, 57, 411-416.

https://doi.org/10.1093/occmed/kqm071

Sjöström, N., Waern, M., \& Hetta, J. (2007). Nightmares and Sleep Disturbances in Relation to Suicidality in Suicide Attempters. Sleep, 30, 91-95.

https://doi.org/10.1093/sleep/30.1.91

Slottje, P., Witteveen, A. B., Twisk, J. W. R., Smidt, N., Huizink, A. C., Mechelen, W. V., \& Smid, T. (2008). Post-Disaster Physical Symptoms of Firefighters and Police Officers: Roles of Types of Exposure and Posttraumatic Stress Symptoms. British Journal of Health Psychology, 13, 327-342. https://doi.org/10.1348/135910707X198793

Stanley, I. H., Hom, M. A., \& Joiner, T. E. (2016). A Systematic Review of Suicidal Thoughts and Behaviors among Police Officers, Firefighters, EMTs, and Paramedics. Clinical Psychology Review, 44, 25-44. https://doi.org/10.1016/j.cpr.2015.12.002

Stanley, I. H., Hom, M. A., Hagan, C. R., \& Joiner, T. E. (2015). Career Prevalence and Correlates of Suicidal Thoughts and Behaviors among Firefighters. Journal of Affective Disorders, 187, 163-171. https://doi.org/10.1016/j.jad.2015.08.007

Stephens, C., Long, M., \& Miller, I. (1997). The Impact of Trauma and Social Support on Posttraumatic Stress Disorder: A Study of New Zealand Police Officers. Journal of Criminal Justice, 25, 303-314. https://doi.org/10.1016/S0047-2352(97)00015-9

Thurnell-Read, T., \& Parker, A. (2008). Men, Masculinities and Firefighting: Occupational Identity, Shop-Floor Culture and Organisational Change. Emotion, Space and Society, 1, 127-134. https://doi.org/10.1016/j.emospa.2009.03.001

Varvel, S. J., Yuhong, H., Shannon, J. K., Tager, D., Bledman, A., Chaichanasakul, A., Mendoza, M., \& Mallinckrodt, B. (2007). Multidimensional Threshold Effects of Social Support in Firefighters: Is More Support Invariably Better? Journal of Counseling Psychology, 54, 458-465. https://doi.org/10.1037/0022-0167.54.4.458

Vogel, D. L., Wade, N. G., \& Ascheman, P. L. (2009). Measuring Perceptions of Stigmatization by Others for Seeking Psychological Help: Reliability and Validity of a New Stigma Scale with College Students. Journal of Counseling Psychology, 56, 301. https://doi.org/10.1037/a0014903 
Vogel, D. L., Wade, N. G., \& Haake, S. (2006). Measuring the Self-Stigma Associated with Seeking Psychological Help. Journal of Counseling Psychology, 53, 325.

https://doi.org/10.1037/0022-0167.53.3.325

Wlodyka, A. (2017). An Examination of Police Stressors and Attitudes towards Seeking Psychological Help. Doctoral Dissertation, Vancouver: University of British Columbia. 